\title{
LA ESTRUCTURA LÓGICA DE LA TEORÍA DEL VALOR TRABAJO
}

\author{
Adolfo García de la Sienra \\ Instituto de Filosofía \\ Facultad de Economía \\ Universidad Veracruzana \\ asienrag@gmail.com
}

RESUMEN: La finalidad de este artículo es proveer una reconstrucción lógica del elemento teórico básico de la teoría del valor trabajo desde la perspectiva de la metateoría estructuralista. Las metas son esbozar con alguna precisión la ley fundamental de la teoría - la ley del valor- así como la aserción empírica.

PALABRAS CLAVE: teoría económica, ley del valor, principio guía, concepción estructuralista

SUMMARY: The aim of the present paper is to provide a logical reconstruction of the basic theory-element of the labor theory of value from the perspective of the structuralist view of theories. The goals are to draft with some precision the fundamental law of the theory - the law of value - as well as the empirical claim.

KEY WORDS: economic theory, law of value, guiding principle, structuralist view

\section{Introducción}

La finalidad central de este trabajo es esbozar con alguna precisión la ley fundamental o principio guía ${ }^{l}$ de la teoría del valor trabajo (TVT), la que habría de definir su elemento teórico básico, desde la perspectiva de la metateoría estructuralista. Como lo señaló Álvarez (1986), esta ley es precisamente la ley del valor. Grosso modo, el sentido de esta ley es que el movimiento de los precios "observados", determinado por las preferencias y la ley de la oferta y la demanda, "oscila" (Capital, vol. 3, p. 293) alrededor del sistema de precios de producción, el cual está determinado, en la esfera de la producción, por el trabajo: cuando el tiempo de trabajo requerido para la producción de un bien cae, su precio (relativo) cae; y cuando se eleva, su precio se eleva ceteris paribus (cfr. Capital, vol. 3, p. 277). De manera que las variaciones de los precios debidas a cualquier factor que no sea un cambio en la estructura de la producción (la tecnología), en la productividad del trabajo o en la ponderación social de los tipos de

${ }^{1}$ Para los conceptos metateóricos aquí empleados, remítase el lector a Balzer, Moulines y Sneed 1987. En particular, para el concepto de principio guía, véase Moulines 1978. 
trabajo concreto, deben ser consideradas como circunstanciales: los precios deben nivelarse en torno al valor expresado en el precio de producción.

$\mathrm{Al}$ hacer esta reconstrucción sistemática de la teoría del valor trabajo, mi intención no es mostrar la aplicabilidad de la metateoría estructuralista a un caso más; para mí está bien claro que esta metateoría es esencialmente correcta y aplicable a las teorías de alta abstracción en economía. Mi intención es más bien clarificar el sentido económico - empírico - del concepto de trabajo abstracto. ¿Qué fenómeno, proceso o estructura social representa dicho concepto? En lo que he publicado antes ${ }^{2}$ me he limitado a demostrar la existencia de representaciones del trabajo abstracto, o a entablar discusiones generales sobre el significado de este concepto, pero no he discutido la ley fundamental y menos la forma de la aserción empírica. Ahora tengo una mejor comprensión del sentido del trabajo abstracto, por ende de la estructura de la teoría, y por eso he regresado a la misma.

Después de introducir el marco conceptual necesario, a partir de la idea de estructura productiva (en la sección 2), procedo a definir el concepto de trabajo abstracto y a demostrar - en condiciones que no son indebidamente restrictivas - la existencia de sistemas de precios que representan el trabajo abstracto en la esfera del mercado (sección 3). En la sección 4 discuto la ley del valor y defino nociones típicas de la teoría. En la sección 5 discuto el significado empírico de la reducción estándar y del trabajo abstracto. La conclusión es que dicha reducción, y por ende el valor trabajo, es la consecuencia involuntaria de un proceso cataláctico en el que interviene una multitud de elecciones individuales, incluyendo las que tienen que ver con el arbitraje bursátil. En la sección 6 caracterizo los modelos potenciales parciales de la teoría y, para motivar la introducción del elemento teórico básico, presento (en la sección 7) el modelo de Leontief. ${ }^{3}$ Finalmente, en la sección 8 expongo los modelos de la teoría formulando la ley fundamental y esbozando la forma general de su aserción empírica.

La teoría aquí formulada forma un cuerpo sólido y consistente de teoría económica que debe ser distinguido y separado de las disquisiciones éticas de Marx sobre el valor. Marx debe ser visto simplemente como un pionero de esta teoría, pues sus formulaciones son más bien oscuras, adolecen de rigor lógico y matemático, y

${ }^{2}$ Véanse García de la Sienra 1980, 1982, 1988, 1992, 1996, 2003a, 2003b, 2007.

${ }^{3}$ El modelo que Marx esboza en el primer tomo de El capital es una variante simplificada de este modelo, con trabajo homogéneo. 
están entremezcladas con consideraciones éticas y políticas que deben quedar excluidas de la teoría económica propiamente dicha. ${ }^{4}$ La TVT no tiene implicaciones revolucionarias ni antirrevolucionarias, ya que es más bien una teoría acerca de la determinación de los sistemas de precios en economías capitalistas. Qué rango de aplicación posee esta teoría es una cuestión enteramente empírica que debe ser tratada con métodos econométricos.

\section{La estructura productiva}

Una estructura productiva es un sistema de procesos de producción más o menos integrados. Es usual suponer que los procesos productivos de un mismo país poseen un grado de integración máximo, pero bien puede suceder, y sucede, que las cadenas productivas tengan un grado débil de integración y que los insumos productivos sean importados. Para fijar ideas, el lector se puede imaginar, cuando describamos la estructura productiva, que es la de la planta industrial de un país sumamente integrado.

Un proceso de producción es un proceso en el que cierta fuerza de trabajo provee un servicio o transforma ciertos objetos en un producto final. En el proceso de producción se distingue la fuerza de trabajo, conceptualizada como una lista (vector) de cantidades de trabajos heterogéneos $y_{1}, \ldots, y_{I}$, medidos en horas/hombre. El número $y_{i}$ denota cierta cantidad de horas de trabajo del tipo $i$ (por ejemplo, de soldador). $I$ es el número de oficios diferentes en la economía. Como es usual, si no se emplean obreros del oficio $y_{i}$ en el proceso, $y_{i}=0$. Se supone que en la estructura productiva hay $L$ tipos distintos de bienes. Aunque en un proceso algunos bienes no aparezcan ni como inputs (insumos) ni como outputs (productos), de todas formas se supondrá que el vector de los primeros y, al igual que el de los segundos $\overline{\mathbf{y}}$, es un vector de dimensión $L$, y que aquellas coordenadas de inputs o outputs que no aparecen en el proceso son iguales a cero.

DEFINICIÓN 1. Un proceso de producción en la versión stock es un

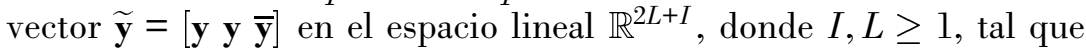
el vector de dimensión $I \mathbf{y}$, así como los vectores de dimensión $L \underline{\mathbf{y}}$

\footnotetext{
${ }^{4}$ Para una amplia e informada discusión de estas consideraciones, véase McCarthy 1990. Desde luego, si la teoría del valor de Marx es una teoría ética, entonces la teoría aquí reconstruida no es la teoría del valor de Marx, pero eso es irrelevante para los fines del presente trabajo.
} 
$\mathrm{y} \overline{\mathbf{y}}$, son todos no negativos. El vector $\mathbf{y}$ se conoce como vector de insumos o inputs laborales, o de trabajo, y es el vector de medios de producción o vector input, y $\overline{\mathbf{y}}$ es el vector producto o vector output. La diferencia $\widehat{\mathbf{y}}=\overline{\mathbf{y}}-\mathbf{y}$ se denomina el output neto o netput del proceso $\widetilde{\mathbf{y}}$. Un proceso de producción en la versión flujo es un vector $\check{\mathbf{y}}=[-\mathbf{y} \widehat{\mathbf{y}}]$, donde $\mathbf{y}$ es el vector de insumos laborales de un proceso en versión stock y $\widehat{\mathbf{y}}$ es el output neto del mismo. Daremos por entendido que $\check{\mathbf{y}}$ es la versión flujo del proceso stock $\tilde{\mathbf{y}}$.

No todos los procesos de producción son igualmente eficientes. Una tecnología eficiente se caracteriza por producir más o la misma cantidad de producto neto usando menos o la misma cantidad de inputs laborales y materiales. Dicho con más precisión.

DEFINICIÓN 2. Sea $Y$ un conjunto de procesos de producción y $\widetilde{\mathbf{y}}, \widetilde{\mathbf{y}}^{\prime}$ elementos de $Y$. Decimos que el proceso $\widetilde{\mathbf{y}}$ es más eficiente que el proceso $\widetilde{\mathbf{y}}^{\prime}$ syss $\check{\mathbf{y}} \geqq \check{\mathbf{y}}^{\prime}$. Es estrictamente más eficiente syss $\check{\mathbf{y}} \geq \check{\mathbf{y}}^{\prime}$. Un proceso $\widetilde{\mathbf{y}} \in Y$ es eficiente syss no existe un proceso en $Y$ estrictamente más eficiente que $\tilde{\mathbf{y}}$.

El gasto de trabajo de cualquier tipo en un proceso eficiente se caracteriza por ser socialmente necesario; es decir, por funcionar bajo condiciones normales, por poseer el grado promedio de habilidad, destreza y velocidad prevaleciente en ese oficio, y por evitar el desperdicio de los materiales e instrumentos de trabajo. ${ }^{5}$

Es común en teoría económica representar las posibilidades tecnológicas de un productor como cierto conjunto de procesos de producción y la elección del productor como un punto de ese conjunto. Supondremos que hay $J$ productores y denotaremos el conjunto de posibilidades tecnológicas del productor $j$ como $Y_{j}(1 \leq j \leq J)$. Productores con la misma tecnología serán tratados como un mismo productor. Admitimos la posibilidad de técnicas alternativas, de manera que un mismo tipo de output puede ser producido por diferentes tecnologías (productores). Esto supone que en general hay menos tipos de outputs producidos que procesos de producción operando, de modo que $L \leq J$. Supondremos que los outputs positivos netos de un productor $j$ son ocasionalmente más de uno, lo cual significa que también admitimos producción conjuntiva (como cuero junto con carne en la industria pecuaria), pero suponemos que ésta no es tan significativa como para revertir la desigualdad $L \leq J$. De igual

\footnotetext{
${ }^{5}$ Marx plantea la definición de tiempo de trabajo socialmente necesario principalmente en El capital. Véase Capital, vol. 1, p. 303.
} 
manera, como los mismos oficios se repiten en diferentes procesos de producción, supondremos que hay más productores que oficios, de modo que también $I \leq J$. Finalmente, supondremos que ningún proceso en $Y_{j}$ se puede obtener tecnológicamente sumando procesos de los otros productores. Esto traerá la consecuencia de que tanto los inputs de trabajo como los netputs de los procesos elegidos por los diferentes productores formarán siempre conjuntos linealmente independientes. Supondremos que $Y_{j}$ es un cono convexo. Como los elementos de $Y_{j}$ son producciones posibles para el $j$-ésimo productor, el conjunto de producción total $Y=\sum_{j=1}^{m} Y_{j}$ está definido. También haremos las suposiciones realistas de que en los procesos de producción representados por los $Y_{j}$ el trabajo es indispensable, y que tanto el trabajo como el sistema mismo son productivos. Precisamos estos conceptos en la siguiente definición.

DEFINICIÓN 3. Y es una estructura productiva syss hay enteros positivos $J, L$ e $I$ tales que, para cada $j=1, \ldots, J$,

(0) $\mathfrak{Y}=\left\langle Y_{1}, \ldots, Y_{J}\right\rangle$;

(1) $Y_{j}$ es un cono convexo cerrado en el espacio lineal $\mathbb{R}^{2 L+I}$;

(2) cada elemento de $Y_{j}$ es un proceso de producción;

(3) no hay más netputs ni oficios que productores, de modo que $L \leq J, I \leq J$, ningún producto neto positivo en $Y_{j}$ se puede obtener tecnológicamente sumando procesos de los otros productores, y ningún vector de inputs de trabajo de un productor se puede obtener sumando inputs de los otros; es decir, cualquier familia $\left\{\mathbf{y}_{1}, \ldots, \mathbf{y}_{J}\right\}$ de inputs de trabajo de los diferentes productores, al igual que cualquier familia $\left\{\widehat{\mathbf{y}}_{1}, \ldots, \widehat{\mathbf{y}}_{J}\right\}$ de netputs de los diferentes productores, es linealmente independiente;

(4) el trabajo es indispensable; i.e., $\mathbf{y}=\mathbf{0}$ implica $\underline{\mathbf{y}}=\underline{\mathbf{0}}$ у $\overline{\mathbf{y}}=\overline{\mathbf{0}}$ para cualquier proceso $[\mathbf{y} \underline{\mathbf{y}} \overline{\mathbf{y}}] \in Y_{j}$;

(5) el trabajo es productivo; i.e., $\underline{\mathbf{y}}=\underline{\mathbf{0}}$ o $\overline{\mathbf{y}}=\overline{\mathbf{0}}$ implica $\mathbf{y}=\mathbf{0}$ para cualquier proceso $[\mathbf{y}, \underline{\mathbf{y}}, \overline{\mathbf{y}}] \in \overline{Y_{j}}$;

(6) $\mathfrak{Y}$ es productiva; es decir, $Y$ contiene un proceso $\widetilde{\mathbf{y}}$ con output neto positivo: $\widehat{\mathbf{y}}>\widehat{\mathbf{0}}$. 
Una tecnología determinada para el productor $j$ se puede representar con el rayo generado por cierto proceso $\widetilde{\mathbf{y}}_{j} \in Y_{j}:\left\{a \widetilde{\mathbf{y}}_{j} \mid a \geq 0\right\}$. Este rayo incluye todos los procesos de producción que son escalamientos de $\widetilde{\mathbf{y}}_{j}$. Por una estructura productiva determinada entendemos el cono polihédrico convexo $K$ que las tecnologías elegidas por los productores generan; es decir, la cápsula convexa de los rayos $\left\{a \widetilde{\mathbf{y}}_{j} \mid a \geq 0\right\}$. Es posible demostrar, sobre la base de las condiciones de la definición 3 , que cada productor puede elegir un proceso de producción $\widetilde{\mathbf{y}}_{j}$ que es eficiente; éste es el sentido de nuestro primer teorema, cuya demostración requiere el siguiente lema previo.

LEMA 1. Sea $Y^{+}$el conjunto de procesos globales con outputs netos no negativos de una estructura productiva $\mathfrak{Y}$. Para todo proceso $\tilde{\mathbf{y}} \in Y^{+}$hay un proceso eficiente $\widetilde{\mathbf{y}}^{*} \in Y^{+}$tal que $\widehat{\mathbf{y}}^{*} \geqq \widehat{\mathbf{y}} y \mathbf{y}^{*} \leqq \mathbf{y}$.

Demostración: La estrategia de la demostración es simple. Mostraré, primero, que el conjunto

$$
E(\widetilde{\mathbf{y}})=\left\{\widetilde{\mathbf{y}}^{\prime} \mid \widehat{\mathbf{y}}^{\prime} \geqq \widehat{\mathbf{y}} \quad \text { y } \quad \mathbf{y}^{\prime} \leqq \mathbf{y}\right\}
$$

es compacto y, segundo, usaré el hecho de que la función $\varphi: E(\widetilde{\mathbf{y}}) \rightarrow$ $\mathbb{R}$, que asigna a cada vector $\widetilde{\mathbf{y}}^{\prime} \in E(\widetilde{\mathbf{y}})$ el número

$$
\left(\widehat{y}_{1}^{\prime}+\cdots+\widehat{y}_{L}^{\prime}\right)-\left(y_{1}^{\prime}+\cdots+y_{I}^{\prime}\right),
$$

es continua. Se seguirá (por el teorema de Weierstrass) que $\varphi$ alcanza un máximo $\widetilde{\mathbf{y}}^{*} \in E(\widetilde{\mathbf{y}})$. Será fácil ver que $\widetilde{\mathbf{y}}^{*}$ es un punto eficiente en $Y^{+}$.

Como $\widetilde{\mathbf{0}} \in Y^{+}$, para mostrar que $E(\widetilde{\mathbf{y}})$ es acotado obsérvese que el conjunto

$$
G=\left\{\mathbf{y}^{\prime} \mid\left[\mathbf{y}^{\prime} \underline{\mathbf{y}}^{\prime} \overline{\mathbf{y}}^{\prime}\right] \in E(\widetilde{\mathbf{y}})\right\}
$$

es acotado, pues $\mathbf{0} \leq \mathbf{y}^{\prime} \leq \mathbf{y}$ para todo $\left[\mathbf{y}^{\prime} \underline{\mathbf{y}}^{\prime} \overline{\mathbf{y}}^{\prime}\right] \in E(\widetilde{\mathbf{y}})$. Ahora bien, si $E(\widetilde{\mathbf{y}})$ no fuera acotado, habría una secuencia $\left(\widetilde{\mathbf{y}}_{k}^{\prime}\right)$ en $E(\widetilde{\mathbf{y}})$ tal que la secuencia de las normas $\left(\left\|\widetilde{\mathbf{y}}_{k}^{\prime}\right\|\right)$ sería creciente y no acotada. No obstante, se puede ver que la correspondiente secuencia de inputs laborales $\left(\mathbf{y}_{k}^{\prime}\right)$ converge en un límite $\mathbf{y}^{\prime} \geq \mathbf{0}$ (no necesariamente en $G$ ) porque $G$ es acotado. Sea

$$
\widetilde{\mathbf{y}}_{k}^{\prime \prime}=\left(\left\|\widetilde{\mathbf{y}}_{k}^{\prime}\right\|\right)^{-1} \widetilde{\mathbf{y}}_{k}^{\prime}
$$


Como $Y^{+}$es un cono, $\widetilde{\mathbf{y}}_{k}^{\prime \prime} \in Y^{+}$. Más aún, $\left(\widetilde{\mathbf{y}}_{k}^{\prime \prime}\right)$ es acotada porque $\left\|\widetilde{\mathbf{y}}_{k}^{\prime \prime}\right\| \leq 1$ para todo $k$. Por ende, podemos afirmar que (una subsecuencia de) $\left(\widetilde{\mathbf{y}}_{k}^{\prime \prime}\right)$ converge en un punto que debe pertenecer a $Y^{+}$ porque $Y^{+}$es cerrado. Como $\left(\left\|\widetilde{\mathbf{y}}_{k}^{\prime \prime}\right\|\right) \rightarrow 1, \widetilde{\mathbf{y}}^{\prime \prime} \neq \widetilde{\mathbf{0}}$ y así, debido a la productividad y a la indispensabilidad del trabajo, $\overline{\mathbf{y}}^{\prime \prime} \geq \overline{\mathbf{0}}$. Por otro lado, como $\left(\mathbf{y}_{k}^{\prime}\right) \rightarrow \mathbf{y}^{\prime}$ conforme $k \rightarrow \infty$,

$$
\begin{aligned}
\mathbf{y}^{\prime \prime} & =\lim _{k \rightarrow \infty} \mathbf{y}_{k}^{\prime \prime} \\
& =\lim _{k \rightarrow \infty}\left(\left\|\tilde{\mathbf{y}}_{k}^{\prime}\right\|+1\right)^{-1} \cdot \lim _{k \rightarrow \infty} \mathbf{y}_{k}^{\prime} \\
& =0 \cdot \mathbf{y}^{\prime} \\
& =\mathbf{0} .
\end{aligned}
$$

Por ende, dado que el trabajo es indispensable, $\overline{\mathbf{y}}^{\prime \prime}=\overline{\mathbf{0}}$. Esta contradicción muestra que $E(\widetilde{\mathbf{y}})$ es acotado.

Ahora bien, es fácil ver que $E(\widetilde{\mathbf{y}})$ es cerrado porque el límite de cualquier secuencia convergente de puntos de $E(\widetilde{\mathbf{y}})$ está en $Y^{+}$-el cual es cerrado - y también satisface la condición para pertenecer a $E(\widetilde{\mathbf{y}})$.

Claramente, como $\varphi$ es continua, tiene un máximo global en un punto $\widetilde{\mathbf{y}}^{*}$ en $E(\widetilde{\mathbf{y}})$. Sostengo que este punto es, en efecto, eficiente en $Y^{+}$, pues supóngase, por el contrario, que hay un $\widetilde{\mathbf{y}}^{* *}$ que es estrictamente más eficiente que $\widetilde{\mathbf{y}}^{*}$, entonces $\varphi\left(\widetilde{\mathbf{y}}^{* *}\right)>\varphi\left(\widetilde{\mathbf{y}}^{*}\right)$, lo cual es imposible porque $\varphi$ ha alcanzado un máximo en $\widetilde{\mathbf{y}}^{*}$.

Suponiendo que los productores han elegido los procesos $\widetilde{\mathbf{y}}_{1}, \ldots, \widetilde{\mathbf{y}}_{J}$, los inputs de trabajo de los procesos se pueden ver como las columnas de la matriz

$$
\mathbf{Y}=\left[\begin{array}{ccc}
y_{11} & \cdots & y_{1 J} \\
\vdots & \ddots & \vdots \\
y_{I 1} & \cdots & y_{I J}
\end{array}\right]
$$

donde el vector $\left[y_{1 j} \cdots y_{I j}\right]^{\mathrm{T}}$ representa los inputs de trabajo del proceso $\tilde{\mathbf{y}}_{j}$. De manera análoga, los inputs materiales se pueden ver como las columnas de

$$
\underline{\mathbf{Y}}=\left[\begin{array}{ccc}
\underline{y}_{11} & \cdots & \underline{y}_{1 J} \\
\vdots & \ddots & \vdots \\
\underline{y}_{L 1} & \cdots & \underline{y}_{L J}
\end{array}\right]
$$


y los outputs como las columnas de

$$
\overline{\mathbf{Y}}=\left[\begin{array}{ccc}
\bar{y}_{11} & \cdots & \bar{y}_{1 J} \\
\vdots & \ddots & \vdots \\
\bar{y}_{L 1} & \cdots & \bar{y}_{L J}
\end{array}\right]
$$

Por ende, la matriz de outputs netos es

$$
\widehat{\mathbf{Y}}=\overline{\mathbf{Y}}-\underline{\mathbf{Y}}=\left[\begin{array}{ccc}
\widehat{y}_{11} & \cdots & \widehat{y}_{1 J} \\
\vdots & \ddots & \vdots \\
\widehat{y}_{L 1} & \cdots & \widehat{y}_{L J}
\end{array}\right]
$$

Cualquier nivel de operación simultánea de los procesos se puede expresar en versión stock como el proceso

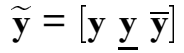

$$
\begin{aligned}
& =\left[\sum_{j=1}^{J} a_{j} \mathbf{y}_{j} \sum_{j=1}^{J} a_{j} \underline{\mathbf{y}}_{j} \sum_{j=1}^{J} a_{j} \overline{\mathbf{y}}_{j}\right] \\
& =\left[\begin{array}{lll}
\mathbf{Y a} & \underline{\mathbf{Y a}} & \mathbf{Y} \\
\mathbf{a}
\end{array}\right] \text {. }
\end{aligned}
$$

En versión flujo o netput como el proceso

$$
\begin{aligned}
\check{\mathbf{y}} & =[-\mathbf{y} \widehat{\mathbf{y}}] \\
& =\left[-\sum_{j=1}^{J} a_{j} \mathbf{y}_{j} \sum_{j=1}^{J} a_{j} \widehat{\mathbf{y}}_{j}\right] \\
& =[-\mathbf{Y a} \widehat{\mathbf{Y}} \mathbf{a}],
\end{aligned}
$$

donde $\mathbf{a}=\left[a_{1} \cdots a_{J}\right] \geqq \mathbf{0}$ es un "estado" o "activación" de la estructura productiva. El conjunto $\{\mathbf{Y a} \mid \mathbf{a} \geqq \mathbf{0}\}=\left\{\mathbf{y} \mid\left[\begin{array}{ll}\mathbf{y} & \mathbf{y}\end{array}\right] \in K\right\}$ de los inputs laborales de $K$ se denotará como $T$; el de los productos netos $\{\widehat{\mathbf{Y}} \mathbf{a} \mid \mathbf{a} \geqq \mathbf{0}\}=\{\widehat{\mathbf{x}} \mid[\mathbf{y} \underline{\mathbf{y}} \overline{\mathbf{y}}] \in K\}$, como $P$.

TEOREma 1. Sea $\mathfrak{Y}$ una estructura productiva. Entonces existe un número finito de actividades $\widetilde{\mathbf{y}}_{1}, \ldots, \widetilde{\mathbf{y}}_{J}$, una de cada productor, tal que toda combinación lineal no negativa de estas actividades es eficiente. 
Demostración: Sea $\widetilde{\mathbf{y}}$ un proceso global eficiente cuyo output neto es positivo, $\widehat{\mathbf{y}}>\widehat{\mathbf{0}}$, y sea $\left\{\widetilde{\mathbf{y}}_{1}, \ldots, \widetilde{\mathbf{y}}_{J}\right\}$ una familia de procesos tales que $\widetilde{\mathbf{y}}=\sum_{k=1}^{J} \widetilde{\mathbf{y}}_{j}$. Mostraré que el cono convexo $K$ generado por estos procesos es un conjunto de actividades eficientes. Si $\mathbf{Y}$ es la matriz de inputs laborales correspondiente a los procesos $\widetilde{\mathbf{y}}_{1}, \ldots, \widetilde{\mathbf{y}}_{J}$, y $\widehat{\mathbf{Y}}$ la correspondiente a los netputs, el proceso $\tilde{\mathbf{y}}$ se puede escribir en versión flujo como $\check{\mathbf{y}}=[-\mathbf{Y a}, \widehat{\mathbf{Y}} \mathbf{a}]$ para cierta activación $\mathbf{a}>\mathbf{0}$.

Supóngase que existe un elemento ineficiente $\widetilde{\mathbf{y}}^{\prime}$ de $K$, de modo que $\check{\mathbf{y}}^{\prime}=\left[-\mathbf{Y} \mathbf{a}^{\prime}, \widehat{\mathbf{Y}} \mathbf{a}^{\prime}\right]$ para alguna activación $\mathbf{a}^{\prime} \geq \mathbf{0}$. Por el lema 1 existe un proceso eficiente $\check{\mathbf{y}}^{*}$ tal que $\widehat{\mathbf{y}}^{*} \geqq \widehat{\mathbf{y}}^{\prime}$ y $\mathbf{y}^{*} \leqq \mathbf{y}^{\prime}$, donde al menos una de estas desigualdades es estricta; i.e. $\hat{\mathbf{y}}^{*} \geq \widehat{\mathbf{y}}^{\prime}$ o $\mathbf{y}^{*} \leq \mathbf{y}^{\prime}$.

Sean $\mathbf{y}^{\prime \prime}=(1-\alpha) \mathbf{Y a}+\alpha \mathbf{Y} \mathbf{a}^{\prime}, \mathbf{y}^{\prime \prime}=(1-\alpha) \underline{\mathbf{Y}} \mathbf{a}+\alpha \underline{\mathbf{Y}} \mathbf{a}^{\prime}$, y $\overline{\mathbf{y}}^{\prime \prime}=$ $(1-\alpha) \overline{\mathbf{Y}} \mathbf{a}+\alpha \overline{\mathbf{Y}} \mathbf{a}^{\prime}$ para $\alpha<0 . \bar{S}_{i} \alpha$ es suficientemente pequeño, $\mathbf{a}^{\prime \prime}=(1-\alpha) \mathbf{a}+\alpha \mathbf{a}^{\prime} \geq \mathbf{0}$ es una activación, de modo que

$$
\begin{aligned}
\widetilde{\mathbf{y}}^{\prime \prime} & =\left[\mathbf{y}^{\prime \prime}, \underline{\mathbf{y}}^{\prime \prime}, \overline{\mathbf{x}}^{\prime \prime}\right] \\
& =\left[\mathbf{Y}\left((1-\alpha) \mathbf{a}+\alpha \mathbf{a}^{\prime}\right), \underline{\mathbf{Y}}\left((1-\alpha) \mathbf{a}+\alpha \mathbf{a}^{\prime}\right), \overline{\mathbf{Y}}\left((1-\alpha) \mathbf{a}+\alpha \mathbf{a}^{\prime}\right)\right] \\
& =\left[\mathbf{Y a}^{\prime \prime}, \underline{\mathbf{Y}} \mathbf{a}^{\prime \prime}, \overline{\mathbf{Y}} \mathbf{a}^{\prime \prime}\right]
\end{aligned}
$$

es un proceso en $K$. Sea $\beta=-(\alpha /(1-\alpha))$. Entonces $0<\beta<1$ y

$$
(1-\beta)\left[-\mathbf{y}^{\prime \prime}, \widehat{\mathbf{y}}^{\prime \prime}\right]+\beta\left[-\mathbf{y}^{*}, \widehat{\mathbf{y}}^{*}\right]
$$

es un proceso global. Pero

$$
(1-\beta) \mathbf{y}^{\prime \prime}+\beta \mathbf{y}^{*}=\mathbf{Y a}-\beta\left(\mathbf{y}^{\prime}-\mathbf{y}^{*}\right) \leqq \mathbf{Y a}
$$

porque $\mathbf{y}^{\prime} \geqq \mathbf{y}^{*}$.

Por otra parte,

$$
\begin{aligned}
(1-\beta) \widehat{\mathbf{y}}^{\prime \prime}+\beta \widehat{\mathbf{y}}^{*} & =\frac{1}{1-\alpha}\left((1-\alpha) \widehat{\mathbf{Y}} \mathbf{a}+\alpha \widehat{\mathbf{Y}} \mathbf{a}^{\prime}\right)+\beta \widehat{\mathbf{y}}^{*} \\
& =\widehat{\mathbf{Y}} \mathbf{a}+\frac{\alpha}{1-\alpha} \widehat{\mathbf{Y}} \mathbf{a}^{\prime}+\beta \widehat{\mathbf{y}}^{*} \\
& =\widehat{\mathbf{Y}} \mathbf{a}-\beta \widehat{\mathbf{Y}} \mathbf{a}^{\prime}+\beta \widehat{\mathbf{y}}^{*} \\
& =\widehat{\mathbf{Y}} \mathbf{a}+\beta\left(\widehat{\mathbf{y}}^{*}-\widehat{\mathbf{Y}} \mathbf{a}^{\prime}\right) \\
& =\widehat{\mathbf{Y}} \mathbf{a}+\beta\left(\widehat{\mathbf{y}}^{*}-\widehat{\mathbf{y}}^{\prime}\right) \\
& \geqq \widehat{\mathbf{Y}} \mathbf{a}
\end{aligned}
$$

porque $\widehat{\mathbf{y}}^{*} \geqq \widehat{\mathbf{y}}^{\prime}$. 
Ahora bien, como

$$
\left[\begin{array}{c}
-\left(\mathbf{Y a}-\beta\left(\mathbf{y}^{\prime}-\mathbf{y}^{*}\right)\right) \\
\widehat{\mathbf{Y}} \mathbf{a}+\beta\left(\widehat{\mathbf{y}}^{*}-\widehat{\mathbf{y}}^{\prime}\right)
\end{array}\right] \geqq\left[\begin{array}{c}
-\mathbf{Y a} \\
\widehat{\mathbf{Y}} \mathbf{a}
\end{array}\right]
$$

la suposición de que $\widetilde{\mathbf{y}}^{\prime}$ es estrictamente menos eficiente que $\widetilde{\mathbf{y}}^{*}$ implica que

$$
\left[\begin{array}{c}
-\left(\mathbf{Y a}-\beta\left(\mathbf{y}^{\prime}-\mathbf{y}^{*}\right)\right) \\
\widehat{\mathbf{Y}} \mathbf{a}+\beta\left(\widehat{\mathbf{y}}^{*}-\widehat{\mathbf{y}}^{\prime}\right)
\end{array}\right] \geq\left[\begin{array}{c}
-\mathbf{Y a} \\
\widehat{\mathbf{Y}} \mathbf{a}
\end{array}\right]
$$

Pero esto contradice la suposición de que $\widetilde{\mathbf{y}}$ es eficiente.

Podemos suponer, por lo tanto, que los productores eligen operar una familia de procesos eficientes cuyo producto neto agregado $\widehat{\mathbf{y}}$ es positivo. Nótese que esto no requiere suponer que los empresarios sean "competitivos"; es decir, tomadores de precios.

Krause (1980) llama "sistema conjuntado general", general joint system, a una estructura de la forma $\langle\mathbf{Y}, \underline{\mathbf{Y}}, \overline{\mathbf{Y}}\rangle$ (aunque la escribe como $\langle\underline{\mathbf{Y}}, \overline{\mathbf{Y}}, \mathbf{Y}\rangle)$, y caracteriza la integración productiva de una estructura tal mediante una condición que llama "conexidad". La definición de esta condición, sin embargo, es difícil de interpretar (él mismo reconoce que es "a bit clumsy", véase la p. 130), aunque garantiza la existencia de una reducción estándar de los inputs de trabajo heterogéneo. La reducción estándar induce una familia de sistemas de precios de producción en un sentido que se precisa adelante. Lo que hace falta subrayar ahora es que la explicación de los precios observados se basa en un sistema de precios de producción tal, el cual depende en parte de la estructura del sistema conjuntado.

Desde la perspectiva estructuralista surge la pregunta de hasta qué punto es menester restringir axiomáticamente el dominio de pretendidas aplicaciones de la teoría dando condiciones suficientes para que el sistema sea conectado. Tal vez el concepto krausiano de conexión sea demasiado restrictivo y deje fuera algunos sistemas que no son conectados en dicho sentido y para los que, sin embargo, existe una reducción estándar. Conforme al método estructuralista, lo que procede es dar condiciones naturales para definir los sistemas conjuntados sin pretender encontrar condiciones suficientes para la existencia de una reducción estándar, aunque esas condiciones (si se les agrega condiciones especiales) permitan demostrar la existencia de alguna reducción tal.

Más bien, la ley general de la teoría debe decir algo así como que, para cualquier sistema de precios de producción en un sistema 
conjuntado, existe una reducción estándar que induce ese sistema de precios de producción, en torno a los cuales oscilan los precios efectivamente observados en el mercado. Lo pertinente para una reconstrucción estructuralista de esta ley es, por lo tanto, introducir el mínimo aparato conceptual requerido para la misma, y mostrar que cualquier reducción estándar induce un único sistema de precios de producción, salvo transformaciones de similaridad. Otro problema es caracterizar el concepto de oscilación de precios.

\section{El concepto de trabajo abstracto}

De manera análoga a como un sistema de precios $\mathbf{p}=\left[p_{1} \cdots p_{L}\right]$ expresa las proporciones de intercambio entre los diferentes bienes y servicios de la estructura económica, mediante la comparación de cada unidad de cualquiera de ellos con un numerario, en cierto proceso social se podrían equiparar las unidades de diferentes trabajos concretos con un estándar (digamos, trabajo de zapatería), y establecer que una hora de trabajo de cierto tipo pesa tanto como $y$ horas de zapatería. Esto expresaría la importancia relativa que una sociedad concede a los distintos oficios en un momento dado.

Esta comparación entre los diferentes gastos de trabajo concreto se puede llevar a cabo mediante una especie de sistema de "salarios sombra" o reducción de los trabajos heterogéneos a una medida común. Por ejemplo, si introducimos el trabajo de zapatería como estándar, podemos comparar todos los gastos de trabajo con el de zapatería mediante un vector positivo $\mathbf{r}=\left[r_{1} \ldots r_{I}\right]$. Sin embargo, no cualquier reducción es admisible sino sólo aquellas que preserven las cantidades de bienes en el sentido de la siguiente definición.

DEFINICIÓN 4. La reducción $\mathbf{r}$ es admisible para $K$ syss $\widehat{\mathbf{y}} \geqq \widehat{\mathbf{y}}^{\prime}$ implica ry $\geq \mathbf{r y}^{\prime}$ y $\widehat{\mathbf{y}} \geq \widehat{\mathbf{y}}^{\prime}$ implica $\mathbf{r y}>\mathbf{r y}^{\prime}$.

Intuitivamente, una reducción admisible se caracteriza por otorgar mayor peso a los inputs de trabajo que producen más. Dada una reducción admisible $\mathbf{r}$, podemos definir una relación de valor sobre el conjunto $P$ de los netputs inducida por $\mathbf{r}$ : decimos que $\widehat{\mathbf{y}}$ representa más valor que $\widehat{\mathbf{y}}^{\prime}$ (módulo r) syss ry $\geq \mathbf{r y}^{\prime}$. Usaremos “ $\widehat{\mathbf{y}} \succcurlyeq \widehat{\mathbf{y}}^{\prime}$ " (" $\widehat{\mathbf{y}} \succ \widehat{\mathbf{y}}^{\prime \prime \prime)}$ para expresar que $\widehat{\mathbf{y}}$ representa no menos (más) valor que $\widehat{\mathbf{y}}^{\prime}$. Tenemos el siguiente resultado.

TEOREma 2. Si r es una reducción admisible para $K$ entonces existe un sistema de precios $\mathbf{p} \geq \mathbf{0}$ tal que, para todo $\widetilde{\mathbf{y}}, \widetilde{\mathbf{y}}^{\prime} \in K$, 
$\mathbf{p} \widehat{\mathbf{y}} \geq \mathbf{p} \widehat{\mathbf{y}}^{\prime}$ syss $\widehat{\mathbf{y}} \succcurlyeq \widehat{\mathbf{y}}^{\prime}$. Este sistema es único salvo transformaciones de similaridad.

Demostración: Sea $\mathbf{t}=\left[\mathbf{r y}_{1} \cdots \mathbf{r y}_{J}\right]$. Si las desigualdades

$$
\widehat{\mathbf{Y}} \mathbf{a} \geqq \mathbf{0}, \quad \mathbf{t a}<0
$$

no tienen solución, entonces $\mathbf{p} \widehat{\mathbf{Y}}=\mathbf{t}$ tiene una solución no negativa $\mathbf{p}$ (cfr. Gale 1960, p. 44).

$\mathrm{Si}$ el vector de insumos laborales $\mathbf{y}_{j}$ fuese igual a cero, la indispensabilidad del trabajo implicaría que el correspondiente vector netput $\widehat{\mathbf{y}}_{j}$ también sería nulo, de modo que el productor $j$ no estaría contribuyendo nada al vector netput global $\widehat{\mathbf{y}}$, el cual seguiría siendo positivo si elimináramos el proceso $\widetilde{\mathbf{y}}$. Es por ello que sin pérdida de generalidad podemos considerar que todos los vectores de inputs laborales son semipositivos.

Ahora bien, ningún vector a que sea solución de $\widehat{\mathbf{Y}} \mathbf{a} \geqq \mathbf{0}$ puede serlo de ta $<0$. Supongamos que a lo es. Entonces a no puede ser no negativo porque, al ser t positivo, tendríamos ta $\geq 0$. Así que a tendría que tener al menos una entrada negativa. Sin pérdida de generalidad, supongamos que $a_{1}, \ldots, a_{k}$ son las entradas no negativas y $a_{k+1}, \ldots, a_{J}$ son las negativas. Como, por hipótesis,

$$
a_{1} \widehat{\mathbf{y}}_{1}+\cdots+a_{k} \widehat{\mathbf{y}}_{k}+a_{k+1} \widehat{\mathbf{y}}_{k+1}+\cdots+a_{J} \widehat{\mathbf{y}}_{J}=\widehat{\mathbf{Y}} \mathbf{a} \geqq \mathbf{0},
$$

se sigue que

$$
a_{1} \widehat{\mathbf{y}}_{1}+\cdots+a_{k} \widehat{\mathbf{y}}_{k} \geqq\left(-a_{k+1}\right) \widehat{\mathbf{y}}_{k+1}+\cdots+\left(-a_{J}\right) \widehat{\mathbf{y}}_{J},
$$

donde $\left[a_{1} \cdots a_{k} \quad 0 \cdots 0\right]$ y $\left[0 \cdots 0-a_{k+1}, \ldots,-a_{J}\right]$ son activaciones del sistema. Como todos los procesos productivos del sistema son eficientes, debemos tener

$$
a_{1} \mathbf{y}_{1}+\cdots+a_{k} \mathbf{y}_{k} \geqq\left(-a_{k+1}\right) \mathbf{y}_{k+1}+\cdots+\left(-a_{J}\right) \mathbf{y}_{J},
$$

pues de lo contrario el proceso $\sum_{j=1}^{k} a_{j} \widetilde{\mathbf{y}}_{j}$ sería estrictamente más eficiente que $\sum_{j=k+1}^{J}\left(-a_{j}\right) \widetilde{\mathbf{y}}_{j}$. Por lo tanto,

$$
\mathbf{r}\left(\sum_{j=1}^{k} a_{j} \mathbf{y}_{j}\right) \geq \mathbf{r}\left(\sum_{j=k+1}^{J}\left(-a_{j}\right) \mathbf{y}_{j}\right)
$$

o 


$$
\mathbf{r}\left(\sum_{j=1}^{J} a_{j} \mathbf{y}_{j}\right) \geq 0
$$

Luego,

$$
\begin{aligned}
\mathbf{t a} & =\left[\begin{array}{lll}
\mathbf{r y}_{1} & \cdots & \mathbf{r y}
\end{array}\right]\left[a_{1} \cdots a_{J}\right]^{\mathrm{T}} \\
& =\sum_{j=1}^{J} a_{j} \mathbf{r} \mathbf{y}_{j} \\
& =\mathbf{r}\left(\sum_{j=1}^{J} a_{j} \mathbf{y}_{j}\right) \\
& \geq 0
\end{aligned}
$$

Para cualesquiera procesos $\widetilde{\mathbf{y}}$ y $\widetilde{\mathbf{y}}^{\prime}$ en $K$, sean a y $\mathbf{a}^{\prime}$ activaciones tales que $\widehat{\mathbf{y}}=[-\mathbf{Y a}, \widehat{\mathbf{Y}} \mathbf{a}]$ y $\widehat{\mathbf{y}}^{\prime}=\left[-\mathbf{Y} \mathbf{a}^{\prime}, \widehat{\mathbf{Y}} \mathbf{a}^{\prime}\right]$. Mostraremos que $\mathbf{p} \widehat{\mathbf{y}} \geq \mathbf{p} \widehat{\mathbf{y}}^{\prime}$ syss $\widehat{\mathbf{y}} \succcurlyeq \widehat{\mathbf{y}}^{\prime}$. Si $\mathbf{p} \geq \mathbf{0}$ es tal que $\mathbf{p} \widehat{\mathbf{Y}}=\left[\mathbf{r y}_{1} \cdots \mathbf{r y}_{J}\right]$ tenemos, para cada productor $j, \sum_{l=1}^{L} p_{l} \widehat{\mathbf{y}}_{l j}=\mathbf{r y}_{j}$. Por lo tanto,

$$
\begin{aligned}
\mathbf{p} \widehat{\mathbf{y}} \geq \mathbf{p} \widehat{\mathbf{y}}^{\prime} & \Leftrightarrow \sum_{j=1}^{J} a_{j} \mathbf{p} \widehat{\mathbf{y}}_{j} \geq \sum_{j=1}^{J} a_{j}^{\prime} \mathbf{p} \widehat{\mathbf{y}}_{j} \\
& \Leftrightarrow \sum_{j=1}^{J} a_{j} \mathbf{r} \mathbf{y}_{j} \geq \sum_{j=1}^{J} a_{j}^{\prime} \mathbf{r} \mathbf{y}_{j} \\
& \Leftrightarrow \mathbf{r}\left(\sum_{j=1}^{J} a_{j} \mathbf{y}_{j}\right) \geq \mathbf{r}\left(\sum_{j=1}^{J} a_{j}^{\prime} \mathbf{y}_{j}\right) \\
& \Leftrightarrow \mathbf{r y} \geq \mathbf{r y} \mathbf{y}^{\prime} \\
& \Leftrightarrow \widehat{\mathbf{y}} \succcurlyeq \widehat{\mathbf{y}}^{\prime} .
\end{aligned}
$$

Para mostrar que $\mathbf{p}$ es único salvo multiplicación por un escalar positivo, demostraremos que la estructura $\langle P, \succcurlyeq,+\rangle$ es una estructura extensiva cerrada.

Nótese, primero, que $\widehat{\mathbf{y}}=\widehat{\mathbf{y}}^{\prime}$ implica que $\widehat{\mathbf{y}} \sim \widehat{\mathbf{y}}^{\prime}$, de manera que $\mathbf{r y}=\mathbf{r y}^{\prime}$. Así, existe una función lineal $\varphi: P \rightarrow \mathbb{R}$ que asigna al netput 
$\widehat{\mathbf{y}}$ el número ry. Con el auxilio de esta función demostraremos que $\succcurlyeq$ es conectada, transitiva, débilmente asociativa y monótona; así como que $\langle P, \succcurlyeq,+\rangle$ satisface la propiedad arquimediana.

$\succcurlyeq$ es conectada.

$$
\begin{aligned}
\widehat{\mathbf{y}}, \widehat{\mathbf{y}}^{\prime} \in P & \Rightarrow \varphi(\widehat{\mathbf{y}}) \geq \varphi\left(\widehat{\mathbf{y}}^{\prime}\right) \vee \varphi\left(\widehat{\mathbf{y}}^{\prime}\right) \geq \varphi(\widehat{\mathbf{y}}) \\
& \Rightarrow \widehat{\mathbf{y}} \succcurlyeq \widehat{\mathbf{y}}^{\prime} \vee \widehat{\mathbf{y}}^{\prime} \succcurlyeq \widehat{\mathbf{y}} .
\end{aligned}
$$

$\succcurlyeq$ es transitiva.

$$
\begin{aligned}
\widehat{\mathbf{y}} \succcurlyeq \widehat{\mathbf{y}}^{\prime} \wedge \widehat{\mathbf{y}}^{\prime} \succcurlyeq \widehat{\mathbf{y}}^{\prime \prime} & \Rightarrow \varphi(\widehat{\mathbf{y}}) \geq \varphi\left(\widehat{\mathbf{y}}^{\prime}\right) \wedge \varphi\left(\widehat{\mathbf{y}}^{\prime}\right) \geq \varphi\left(\widehat{\mathbf{y}}^{\prime \prime}\right) \\
& \Rightarrow \varphi(\widehat{\mathbf{y}}) \geq \varphi\left(\widehat{\mathbf{y}}^{\prime \prime}\right) \\
& \Rightarrow \widehat{\mathbf{y}} \succcurlyeq \widehat{\mathbf{y}}^{\prime \prime}
\end{aligned}
$$

$\succcurlyeq$ es débilmente asociativa.

Esto es probar que $\widehat{\mathbf{y}}+\left(\widehat{\mathbf{y}}^{\prime}+\widehat{\mathbf{y}}^{\prime \prime}\right)=\left(\widehat{\mathbf{y}}+\widehat{\mathbf{y}}^{\prime}\right)+\widehat{\mathbf{y}}^{\prime \prime}$, lo cual es equivalente a la identidad $\varphi\left[\widehat{\mathbf{y}}+\left(\widehat{\mathbf{y}}^{\prime}+\widehat{\mathbf{y}}^{\prime \prime}\right)\right]=\varphi\left[\left(\widehat{\mathbf{y}}+\widehat{\mathbf{y}}^{\prime}\right)+\widehat{\mathbf{y}}^{\prime \prime}\right]$, pero ésta es obvia por la linealidad de $\varphi$.

$\succcurlyeq$ es monótona.

$$
\begin{aligned}
\widehat{\mathbf{y}} \succcurlyeq \widehat{\mathbf{y}}^{\prime} & \Rightarrow \varphi(\widehat{\mathbf{y}}) \geq \varphi\left(\widehat{\mathbf{y}}^{\prime}\right) \\
& \Rightarrow \varphi(\widehat{\mathbf{y}})+\varphi\left(\widehat{\mathbf{y}}^{\prime \prime}\right) \geq \varphi\left(\widehat{\mathbf{y}}^{\prime}\right)+\varphi\left(\widehat{\mathbf{y}}^{\prime \prime}\right) \quad \forall \widehat{\mathbf{y}}^{\prime \prime} \in N \\
& \Rightarrow \widehat{\mathbf{y}}+\widehat{\mathbf{y}}^{\prime \prime} \succcurlyeq \widehat{\mathbf{y}}+\widehat{\mathbf{y}}^{\prime \prime} \quad \forall \widehat{\mathbf{y}}^{\prime \prime} \in N .
\end{aligned}
$$

$\langle P, \succcurlyeq,+\rangle$ satisface la propiedad arquimediana.

Queremos probar que si $\widehat{\mathbf{y}}_{1} \succ \widehat{\mathbf{y}}_{2}$ entonces, para cualesquiera $\widehat{\mathbf{y}}_{3}, \widehat{\mathbf{y}}_{4} \in P$, existe un entero positivo $\nu$ tal que $\nu \widehat{\mathbf{y}}_{1}+\widehat{\mathbf{y}}_{3} \succcurlyeq \nu \widehat{\mathbf{y}}_{2}+\widehat{\mathbf{y}}_{4}$. Escójase cualquier entero positivo $\nu$ que satisfaga la desigualdad

$$
\nu \geq \frac{\varphi\left(\widehat{\mathbf{y}}_{4}\right)-\varphi\left(\widehat{\mathbf{y}}_{3}\right)}{\varphi\left(\widehat{\mathbf{y}}_{1}\right)-\varphi\left(\widehat{\mathbf{y}}_{2}\right)} .
$$

Como $\varphi\left(\widehat{\mathbf{y}}_{1}\right)-\varphi\left(\widehat{\mathbf{y}}_{2}\right)>0$, si $\widehat{\mathbf{y}}_{3} \succcurlyeq \widehat{\mathbf{y}}_{4}$ la desigualdad se satisface con cualquier entero positivo $\nu$. De otro modo, simplemente se toma uno que sea lo suficientemente grande, pues se tiene 


$$
\begin{aligned}
\nu\left[\varphi\left(\widehat{\mathbf{y}}_{1}\right)-\varphi\left(\widehat{\mathbf{y}}_{2}\right)\right] \geq \varphi\left(\widehat{\mathbf{y}}_{4}\right)-\varphi\left(\widehat{\mathbf{y}}_{3}\right) & \Rightarrow \nu \varphi\left(\widehat{\mathbf{y}}_{1}\right)+\varphi\left(\widehat{\mathbf{y}}_{3}\right) \geq \nu \varphi\left(\widehat{\mathbf{y}}_{2}\right)+\varphi\left(\widehat{\mathbf{y}}_{4}\right) \\
& \Rightarrow \varphi\left(\nu \widehat{\mathbf{y}}_{1}\right)+\varphi\left(\widehat{\mathbf{y}}_{3}\right) \geq \varphi\left(\nu \widehat{\mathbf{y}}_{2}\right)+\varphi\left(\widehat{\mathbf{y}}_{4}\right) \\
& \Rightarrow \varphi\left(\nu \widehat{\mathbf{y}}_{1}+\widehat{\mathbf{y}}_{3}\right) \geq \varphi\left(\nu \widehat{\mathbf{y}}_{2}+\widehat{\mathbf{y}}_{4}\right) \\
& \Rightarrow \nu \widehat{\mathbf{y}}_{1}+\widehat{\mathbf{y}}_{3} \succcurlyeq \nu \widehat{\mathbf{y}}_{2}+\widehat{\mathbf{y}}_{4} .
\end{aligned}
$$

Por lo tanto, $\langle P, \succcurlyeq,+\rangle$ es una estructura extensiva cerrada y $\varphi$ es, por construcción, una representación de la misma. Si $\psi: P \rightarrow \mathbb{R}$ es otra representación, entonces existe $\alpha>0$ tal que $\psi=\alpha \varphi$; es decir,

$$
\psi(\widehat{\mathbf{y}})=\alpha \varphi(\widehat{\mathbf{y}})=\alpha(\mathbf{p} \widehat{\mathbf{y}})=(\alpha \mathbf{p}) \widehat{\mathbf{y}}
$$

o, lo que es lo mismo, $\psi$ es la transformación lineal $\alpha \mathbf{p}$. Esto establece que $\mathbf{p}$ es único salvo multiplicación por un escalar positivo.

En términos de los conceptos introducidos hasta ahora es posible proporcionar una definición precisa del elusivo concepto de trabajo abstracto. El trabajo abstracto no es más que la relación entre inputs de trabajo inducida por la reducción, de manera que podemos representarlo como la estructura que se define enseguida.

DEFINICIÓn 5. Sea r una reducción admisible para $K$. Definimos la relación binaria $\succsim$ sobre $T$ (módulo r) mediante la condición

$$
\mathbf{y} \succsim \mathbf{y}^{\prime} \quad \text { syss } \quad \mathbf{r y} \geq \mathbf{r y}^{\prime}
$$

$\langle T, \succsim\rangle$ es llamada trabajo abstracto.

Como es usual, escribimos $\mathbf{y} \sim \mathbf{y}^{\prime}$ si $\mathbf{y} \succsim \mathbf{y}^{\prime}$ y $\mathbf{y}^{\prime} \succsim \mathbf{y} ;$ y $\mathbf{y} \succ \mathbf{y}^{\prime}$ si $\mathbf{y} \succsim \mathbf{y}^{\prime}$ pero no $\mathbf{y}^{\prime} \succsim \mathbf{y}$.

De aquí en adelante consideraremos $K$ y r como fijos. El trabajo abstracto no es más que una comparación entre inputs de trabajo. Lo que hace una reducción es servir de índice de esta comparación. Si a un gerente se le pidiera que evaluara la contribución que cada oficio dentro del proceso de producción aporta al producto neto final, ${ }^{6}$ podría ofrecer una reducción normalizada; es decir, una reducción en el simplex de $\mathbb{R}^{I}$. Esta ponderación diría que el oficio $i(1 \leq i \leq I)$

\footnotetext{
${ }^{6}$ Un problema aún no resuelto es si el concepto de valor de Shapley puede servir para hacer esta evaluación.
} 
aporta la fracción $r_{i}$ del valor total, de manera que $r_{1} \mathbf{p} \widehat{\mathbf{y}}+\cdots+r_{I} \mathbf{p} \widehat{\mathbf{y}}=$ $\left(\sum_{i=1}^{I} r_{i}\right) \mathbf{p} \widehat{\mathbf{y}}=\mathbf{p} \widehat{\mathbf{y}}$.

Examinemos ahora un vector de inputs laborales fijo $\mathbf{y}_{0}$. La cantidad de trabajo abstracto que $\mathbf{y}_{0}$ representa es $\mathbf{r y}_{0}$. Si $y_{i}$ es positivo, $r_{i} y_{i}$ es el peso determinado que la cantidad de trabajo $y_{i}$ del oficio $i$ tiene en el proceso de producción correspondiente. La cantidad de trabajo abstracto contenida en cualquier otro vector de inputs de trabajo y se puede expresar como un múltiplo de $\mathbf{y}_{0}$. En efecto, como $\mathbf{r y}=\mathbf{r}\left(\frac{\mathbf{r y}}{\mathbf{r y} \mathbf{y}_{0}} \mathbf{y}_{0}\right), \mathbf{y} \sim \frac{\mathbf{r y}}{\mathbf{r y} \mathbf{y}_{0}} \mathbf{y}_{0}$, de manera que el vector $\mathbf{y}$ es equivalente a $\frac{\mathbf{r y}}{\mathbf{r y}_{0}}$ unidades del complejo $\mathbf{y}_{0}$. Como $\frac{1}{\mathbf{r y}_{0}} \mathbf{r}$ es también una reducción admisible, de aquí en adelante podemos suponer que $\mathbf{r}$ ha sido ya relativizada a $\mathbf{y}_{0}$ de este modo. Así, ry es la cantidad de trabajo abstracto asociado a $\mathbf{y}$ en términos de $\mathbf{y}_{0}$. Nótese que, en particular, si $\mathbf{y}_{0}$ sólo contiene número positivo en la coordenada correspondiente al trabajo simple no calificado, entonces $\mathbf{r}$ reduce todos los tipos de trabajo a trabajo simple. Sin embargo, podría no existir un vector $\mathbf{y}_{0}$ con estas características.

\section{Valor y precio: la ley del valor}

Para poder discutir el sentido de la ley del valor, necesitamos introducir una serie de nociones previas. Como ya se dijo, consideramos fijo el sistema conjuntado $K$ y una reducción admisible relativizada r. Introducimos el sistema de salarios horarios $\mathbf{w}$, el cual es un vector $\left[w_{1} \cdots w_{I}\right]$, donde $w_{i}$ es la cantidad de dinero que se paga por una hora de servicio del oficio $i(i=1, \ldots, I)$.

Definición 6. Sea p el precio inducido por la reducción r. Definimos, para cualquier activación a del sistema productivo:

(1) El capital variable $v$ es el valor monetario de la fuerza de trabajo operada con intensidad a: $v=$ wYa.

(2) El capital constante $c$ es el valor monetario de los inputs operados con intensidad a: $c=\mathbf{p} \underline{\mathbf{Y a}}$.

(3) El valor monetario del producto neto es $p=\mathbf{p} \widehat{\mathbf{Y}} \mathbf{a}$.

(4) El beneficio total $b$ es la diferencia entre el valor monetario del producto neto y el capital variable: $b=p-v$. 
(5) La tasa promedio de ganancia $r$ es la razón entre el beneficio total $b$ y los costos de capital: $c+v: r=b /(c+v)$.

(6) La tasa máxima de ganancia $r^{*}$ es $r^{*}=p / c$.

Nótese que la tasa máxima tiene lugar cuando el valor del capital variable es igual a 0 .

Sea

$$
\mathbf{B}=\left[\begin{array}{ccc}
b_{11} & \cdots & b_{1 I} \\
\vdots & \ddots & \vdots \\
b_{L 1} & \cdots & b_{L I}
\end{array}\right]
$$

el sistema de consumo de los trabajadores, donde la columna $i$-ésima, la cual suponemos que es un elemento de $P$, es el vector de consumo de los trabajadores del oficio $i$. Así, suponiendo (lo que haremos) que no hay ahorro de los salarios, $w_{i}=\sum_{i=1}^{n} p_{i} b_{i j}$. Para la activación a tenemos $v=\mathbf{p B Y a}$, de modo que, en este caso, el tiempo de trabajo necesario para operar el sistema $K$ con intensidad a (el cual no se debe confundir con el tiempo socialmente necesario requerido para que un operario del oficio $j$ desempeñe una labor) es precisamente $v$. Como el valor añadido al producto neto por la fuerza laboral bajo la activación a no es más que $v$, podemos introducir la siguiente definición.

Definición 7. La tasa de plusvalía bajo la activación a es

$$
e=\frac{\text { valor añadido }- \text { tiempo de trabajo necesario }}{\text { tiempo de trabajo necesario }}=\frac{p-v}{v} .
$$

La relación entre la tasa promedio de ganancia, $r$, y la tasa de plusvalía $e$, se determina del siguiente modo:

$$
\begin{aligned}
r & =\frac{p-v}{c+v} \\
& =\frac{p(p-v)}{p(c+v)} \\
& =\frac{p(p-v)}{v c+p c-v c+p v} \\
& =\frac{(p-v) p}{v c+(p-v) c+v p}
\end{aligned}
$$




$$
\begin{aligned}
& =\frac{(p-v) p}{v c} \cdot \frac{v c}{v c+(p-v) c+v p} \\
& =\frac{e r^{*}}{1+e+r^{*}}
\end{aligned}
$$

Es obvio que $r$ es positiva si, y sólo si, $e$ es positiva, ${ }^{7}$ y que la tasa promedio de ganancia es una función creciente de la tasa de plusvalía.

Hasta aquí el nivel de la tasa de ganancia promedio ha dependido de la activación a. No dependería de ninguna activación en particular si $r$ fuese uniforme, en cuyo caso el sistema de precios $\mathbf{p}$ asumiría la forma de precio de producción; es decir,

$$
\mathbf{p} \overline{\mathbf{Y}}=(1+r)[\mathbf{w} \mathbf{Y}+\mathbf{p} \underline{\mathbf{Y}}] .
$$

De hecho, tenemos las siguientes equivalencias.

TEOREMA 3. Si r es uniforme entonces las siguientes aserciones son equivalentes para toda activación a:

(1) $r^{*}$ es independiente de a.

(2) e es independiente de a.

(3) $\mathbf{w Y} \propto \mathbf{Y} \mathbf{Y}$

Demostración: Supóngase que $r$ es uniforme. Tenemos:

$(1) \Rightarrow(2)$. Supóngase que $r^{*}$ es independiente de a. Como

$$
r=\frac{e r^{*}}{1+e+r^{*}},
$$

se sigue que

$$
e=\frac{r\left(1+r^{*}\right)}{r^{*}-r} .
$$

Dado que el lado derecho de esta ecuación es independiente de la activación a, también debe serlo el izquierdo.

$(2) \Rightarrow(3)$. Supóngase que $e$ es independiente de cualquier activación. Esto significa que $e$ es una constante tal que

${ }^{7}$ Éste es el famoso teorema marxiano fundamental, demostrado por primera vez de manera formal, para modelos específicos de la TVT, por Morishima y Seton (1961) y Okishio (1963). 


$$
\frac{\mathbf{p} \widehat{\mathbf{y}}-\mathbf{w y}}{\mathbf{w y}}=e
$$

para cualquier proceso $\widetilde{\mathbf{y}} \in K$. Como ry $=\mathbf{p} \widehat{\mathbf{y}}$, se sigue que

$$
\mathbf{w y}=\kappa \mathbf{r y}
$$

donde $\kappa=1 /(e+1)$.

$(3) \Rightarrow(1)$. Supóngase que wy $=\kappa \mathbf{r y}$ para algún $\kappa$ y todo $\widetilde{\mathbf{y}} \in K$. Como ry $=\mathbf{p} \widehat{\mathbf{y}}$ y $\mathbf{p}$ es un precio de producción, tenemos

$$
\frac{1}{1+r} \mathbf{p y}=\mathbf{w y}+\mathbf{p y}
$$

Luego,

$$
\begin{aligned}
\frac{1}{1+r} \mathbf{p \overline { y }}-\mathbf{p y} \underline{\mathbf{y}} & =\mathbf{w y} \\
& =\kappa \mathbf{p} \widehat{\mathbf{y}} \\
& =\kappa(\mathbf{p} \overline{\mathbf{y}}-\mathbf{p y})
\end{aligned}
$$

de donde, finalmente,

$$
\frac{1}{1+r} \mathbf{p} \overline{\mathbf{y}}=\mathbf{p y} .
$$

Por ende, si $\lambda^{\prime}=1 /(1+r)$,

$$
\begin{aligned}
e & =\frac{\mathbf{p} \overline{\mathbf{y}}-\mathbf{p y}}{\mathbf{p y}} \\
& =\frac{\mathbf{p} \overline{\mathbf{y}}}{\mathbf{p y}}-1 \\
& =\frac{\overline{\mathbf{p y}}}{\lambda^{\prime} \mathbf{p} \overline{\mathbf{y}}}-1 \\
& =\lambda .
\end{aligned}
$$

donde $\lambda=\left(1 / \lambda^{\prime}\right)-1$.

Introducimos ahora canónicamente la noción, ya discutida, de reducción estándar.

DEFINICIÓN 8. Una reducción admisible $\mathbf{r}$ es una reducción estándar syss, para el sistema de precios $\mathbf{p}$ inducido por $\mathbf{r}, r^{*}$ es uniforme; 
i.e., independiente de cualquier activación a. En otras palabras, syss $\mathbf{p} \widehat{\mathbf{Y}}=r^{*} \mathbf{p} \underline{\mathbf{Y}}$.

Decimos que una reducción estándar está normalizada si pertenece al simplex de $\mathbb{R}^{I}$. Consecuencias inmediatas de esta definición son las siguientes.

TeOrema 4. Si $\mathbf{r}$ es una reducción estándar normalizada entonces e es uniforme $y$
(1) $r=\frac{e r^{*}}{1+e+r^{*}}$.
(2) $\mathbf{w} \mathbf{Y}=\frac{1}{1+e} \mathbf{r Y}$.
(3) $\mathbf{p} \underline{\mathbf{Y}}=\frac{1}{r^{*}} \mathbf{r Y} y \mathbf{p} \overline{\mathbf{Y}}=\frac{1+r^{*}}{r^{*}} \mathbf{r Y}$.

Demostración: Las cláusulas (1) y (2) ya han sido demostradas antes. Claramente, para todo $\widetilde{\mathbf{y}} \in K$,

$$
\frac{1}{r^{*}} \mathbf{r y}=\frac{\mathbf{p y}}{\mathbf{p} \widehat{\mathbf{y}}} \cdot \mathbf{p} \widehat{\mathbf{y}}=\mathbf{p y} .
$$

Además,

$$
\begin{aligned}
\frac{1+r^{*}}{r^{*}} \mathbf{r y} & =\left(1+\frac{\mathbf{p y}}{\mathbf{p y}}\right) \frac{\mathbf{p y}}{\mathbf{p y}} \cdot \mathbf{p} \widehat{\mathbf{y}} \\
& =\mathbf{p y}+\mathbf{p} \widehat{\mathbf{y}} \\
& =\mathbf{p} \overline{\mathbf{y}} . \quad \square
\end{aligned}
$$

Nótese que la parte (3) del teorema 3 y la (2) del teorema 4 significan que, bajo una reducción estándar, el costo salarial total es proporcional al valor de la producción total. Este hecho es relevante para discutir el significado empírico de la reducción estándar, lo cual haremos en la siguiente sección.

\section{El significado empírico de la reducción estándar}

Es un requisito del individualismo metodológico ${ }^{8}$ que los conceptos explicativos en ciencias sociales o "ciencias de la conducta" tengan

${ }^{8}$ El concepto de individualismo metodológico que presupongo aquí se explica en García de la Sienra 2010. 
que ver con las elecciones individuales. Esto exige que las reducciones, en particular las reducciones estándar, sean el resultado de elecciones individuales. Esto no significa que deba haber un gran elector que adopte las reducciones, sino que el proceso que las produce, por complejo que sea, debe ser el resultado de elecciones individuales, aunque dicho resultado no haya sido la meta de ningún agente individual: puede ser una unintended consequence de multitud de elecciones individuales. Pero, en todo caso, ¿cuál es la realidad empírica de una reducción? ¿Es posible señalar alguna entidad en el proceso social de producción que corresponda a una reducción estándar?

La respuesta es positiva si observamos que, como se establece en la parte (3) de la definición 3, ninguna línea de producción de la empresa $k$ se puede obtener "sumando" las de otros productores. Como $s \leq m$, esto implica que las columnas de la matriz $\mathbf{Y}$ generan $\mathbb{R}^{s}$ y por ende $\mathbf{Y}$ tiene un inversa $\mathbf{A}$ por la derecha:

$$
\mathbf{Y A}=\mathbf{I}_{s},
$$

donde las columnas de $\mathbf{I}_{s}$ son los vectores de la base canónica de $\mathbb{R}^{s}$, de modo que, como wY $\propto \mathbf{r Y}$, existe $\alpha>0$ tal que

$$
\mathbf{r}=\mathbf{r} \mathbf{I}_{s}=\mathbf{r Y A}=\alpha \mathbf{w} \mathbf{Y A}=\alpha \mathbf{w} \mathbf{I}_{s}=\alpha \mathbf{w} .
$$

En otras palabras, la reducción es la ponderación de los oficios efectuada por las tasas salariales de "equilibrio", las cuales no necesariamente coinciden con las tasas observadas en un momento del proceso. La fijación de estas tasas es un proceso complejo en el que intervienen sindicatos, patrones, y "el mercado" a través del arbitraje bursátil, que presiona hacia la igualación de la tasa de ganancia. Desde el punto de vista estructuralista, el de reducción es un concepto TVTteórico que sólo puede ser determinado a partir del movimiento de los precios y los salarios, entre muchas otras cosas, presuponiendo la ley del valor.

\section{Los modelos potenciales parciales}

Hemos demostrado que toda reducción estándar induce un sistema admisible de precios de producción. La ley del valor se puede esbozar, en primera aproximación, como la aserción de que los precios observados están determinados por la estructura productiva y una reducción estándar. Los modelos de la teoría quedarían definidos una vez que se precisa esta aserción. ¿Cómo podría precisarse? 
La idea básica y central es que el precio de producción inducido por la reducción estándar vigente es un atractor o sumidero en el sentido de la teoría dinámica. Sin embargo, es conveniente clarificar suficientemente la versión estática de la TVT antes de pasar a consideraciones dinámicas. La forma más sencilla de la ley del valor sería que el precio en el estado vigente es aproximadamente idéntico al precio de producción inducido por alguna reducción estándar. Para poder alcanzar esta formulación necesitamos extender un poco el aparato conceptual, la extensión se introducirá como parte de los modelos potenciales.

En analogía con el concepto de economía de Debreu (1973, pp. 9697), podemos pensar en una economía marxiana como un conjunto de consumidores y productores dotados de espacios de elecciones restringidas por diferentes condicionantes, como la estructura de la tecnología, los recursos totales disponibles, o las distribuciones factibles del trabajo social.

Hemos descrito ya el concepto de estructura de la tecnología. Los recursos totales disponibles a la economía al comienzo del ciclo productivo, ${ }^{9}$ incluyendo los laborales, se pueden representar mediante un vector $I+L$ dimensional $\boldsymbol{\omega}$, como lo hace Debreu (1973, p. 96).

En la teoría del valor neoclásica, cada consumidor posee como espacio de consumo un subconjunto de $\mathbb{R}^{L}$. Así, cada consumidor queda caracterizado por una estructura de preferencia $\langle X, \succsim\rangle$. Marx atribuía a los consumidores obreros una canasta de consumo rígida, pero al menos en los países capitalistas avanzados esa suposición no parece realista y, en todo caso, es innecesariamente restrictiva. De hecho, nada impide suponer que se puede representar a cada obrero como consumidor mediante una estructura de preferencia. Es común suponer que cada obrero se emplea en un solo oficio y que los obreros del mismo oficio tienen todos la misma relación de preferencia, de modo que cualquiera de ellos es "representativo". Sin embargo, antes de garantizar que los obreros pueden maximizar su preferencia, una teoría adecuada del valor debe establecer una condición de subsistencia de los consumidores. Así, otra condición de factibilidad es que la activación de la economía debe garantizar (además de la subsistencia y la ganancia del capitalista) la subsistencia del proletariado. Esta condición, junto con la de que ningún obrero puede trabajar jornadas excesivamente largas, corresponde a la expresada en la suposición II requerida para la demostración del primer teorema de existencia de

\footnotetext{
${ }^{9}$ Se estila, para reafirmar ideas, tomar un año como la duración de este ciclo.
} 
un equilibrio competitivo por Arrow y Debreu (1954), la cual excluye menús de consumo insuficientes para mantener la vida. Estos autores expresan dicha condición diciendo que hay un vector $\boldsymbol{\xi}_{i}$ tal que $\boldsymbol{\xi}_{i} \leqq \mathbf{x}_{i}$ para todo $\mathbf{x}_{i} \in X_{i}$. Aquí suponemos que $\operatorname{los} X_{i}$ son subconjuntos convexos de $\mathbb{R}^{L}$ que satisfacen esta condición.

Así, supondremos que hay $I+1$ consumidores, y que el $I+1$ es el representativo de la "burguesía". Entendemos por una economía un conjunto de consumidores y productores sometidos a la condición restrictiva expresada por $\boldsymbol{\omega}$. De esta manera, una economía se puede representar, à la Debreu, mediante una estructura de la forma

$$
\left\langle\left\langle X_{i}, \succsim_{i}\right\rangle, Y_{j}, \boldsymbol{\omega}\right\rangle
$$

donde $\succsim_{i}$ es un ordenamiento débil de $X_{i}(1 \leq i \leq I+1), Y_{j}$ es el conjunto de posibilidades de producción del productor $j$, y $\boldsymbol{\omega}$ representa los recursos totales de la economía. Formalmente:

Definición 9. E es una economía syss existen $X_{i}, \succsim_{i}, Y_{j}, \boldsymbol{\omega}$ y enteros positivos $I, J$ y $L$, tales que

(0) $\mathfrak{E}=\left\langle\left\langle X_{i}, \succsim_{i}\right\rangle, Y_{j}, \boldsymbol{\omega}\right\rangle$

(1) para cada $i \in\{1, \ldots, I+1\}:\left\langle X_{i}, \succsim_{i}\right\rangle$ es un orden débil, donde $X_{i}$ es un subconjunto convexo del ortante no negativo de $\mathbb{R}^{L}$ acotado por debajo por un vector semipositivo;

(2) $\left\langle Y_{1}, \ldots, Y_{J}\right\rangle$ es una estructura productiva;

(3) $\boldsymbol{\omega}$ es un vector positivo en $\mathbb{R}^{I} \times \mathbb{R}^{L}$.

Una economía puede entrar en distintos estados, representados por los procesos de producción que los empresarios operan, los menús de consumo que los consumidores demandan, y el precio vigente observado p. Esto motiva la siguiente definición.

DEFINICIÓN 10. Un estado de la economía $\mathfrak{E}$ es un vector de la forma

$$
\mathbf{e}=\left(\mathbf{x}_{1}, \ldots, \mathbf{x}_{I}, \mathbf{x}_{I+1}, \tilde{\mathbf{y}}_{1}, \ldots, \tilde{\mathbf{y}}_{J}, \mathbf{p}\right) .
$$

donde $\mathbf{x}_{i} \in X_{i}$ para cada $i \in\{1, \ldots, I+1\}, \widetilde{\mathbf{y}}_{j} \in Y_{j}$ para cada $j \in$ $\{1, \ldots, J\}$, y $\mathbf{p}$ es un vector semipositivo. El estado es realizable syss $\mathbf{x}+\underline{\mathbf{y}} \leq \boldsymbol{\omega}$, donde $\mathbf{x}=\sum_{i=1}^{I+1} \mathbf{x}_{i}$ y $\underline{\mathbf{y}}=\sum_{j=1}^{J} \underline{\mathbf{y}}_{j}$. En particular, un 
estado de la estructura productiva es un vector que sólo incluye los procesos de producción: $\left(\widetilde{\mathbf{y}}_{1}, \ldots, \widetilde{\mathbf{y}}_{J}\right)$.

Lo que he llamado aquí "economía" corresponde a los modelos potenciales parciales de la TVT. Para motivar la introducción de los modelos, en particular la formulación de la ley fundamental, será instructivo considerar el caso particular del modelo de Leontief.

\section{El modelo de Leontief}

El modelo de Leontief representa un estado de la economía en el que cada tipo de bien es producido por un solo proceso (ausencia de técnicas alternativas), no hay producción conjuntiva, y el único factor primario es el trabajo, que se supone que es homogéneo. La matriz $\underline{\mathbf{Y}}$ de insumos es por ello cuadrada de orden $L$, mientras que la matriz de outputs $\overline{\mathbf{Y}}=\mathbf{I}$ es la matriz identidad de orden $L$. Si se supone que el trabajo es homogéneo, la matriz de inputs laborales $\mathbf{Y}$ será un vector columna de $I$ dimensiones; no obstante, aquí seguiremos suponiendo que el trabajo es heterogéneo, de modo que $\mathbf{Y}$ sigue siendo una matriz de orden $I \times J$. Se supone que todas las ramas de la economía están interconectadas, así que la matriz de inputs, $\underline{\mathbf{Y}}$, es indescomponible. $^{10}$

Sea $\mathbf{w}$, el sistema salarial imperante, una reducción estándar y $\mathbf{p}$ el sistema de precios inducido por $\mathbf{w}$. Entonces $\mathbf{p}$ asume la forma de precio de producción:

$$
\mathbf{p I}=(1+r)[\mathbf{w} \mathbf{Y}+\mathbf{p} \underline{\mathbf{Y}}] .
$$

Como $r \geq r^{*} \geq 0, \rho=1 /(1+r)$ está definido y tenemos

$$
\mathbf{p}[\rho \mathbf{I}-\underline{\mathbf{Y}}]=\mathbf{w} \mathbf{Y} .
$$

Como (por el teorema 2) $\mathbf{p} \geq \mathbf{0}, \rho>\lambda^{*}$, donde $\lambda^{*}$ es la raíz de Frobenius de $\underline{\mathbf{Y}} .{ }^{11}$ Por lo tanto, $\rho \mathbf{I}-\underline{\mathbf{Y}}$ es no singular y $[\rho \mathbf{I}-\underline{\mathbf{Y}}]^{-1}$ es una matriz semipositiva. Así que el sistema de precios p se obtiene como una función de la estructura productiva y la reducción w:

$$
\mathbf{p}=[\rho \mathbf{I}-\underline{\mathbf{Y}}]^{-1} \cdot \mathbf{w} \mathbf{Y} .
$$

Ésta es la forma específica que asume la ley del valor en las estructuras de Leontief.

\footnotetext{
${ }^{10}$ Para una definición precisa de este concepto, véase Takayama 1985, p. 370.

${ }^{11}$ Por el teorema 4.D.2 en Takayama 1985, p. 392.
} 


\section{Los modelos de la teoría}

El ejemplo de Leontief sugiere que el sistema de precios es, en efecto, algo así como una modificación nómica del trabajo abstracto, obtenido "durch allgemeine Gesetze bestimmte Modifikationen". ${ }^{12}$ Pero es más exacto decir que el sistema de precios "observado" se aproxima a un precio de producción que es una modificación nómica del estado del sistema productivo junto con alguna reducción estándar. Esto es, el principio guía o ley fundamental estaría indicando que el precio observado $\mathbf{p}$ debe ser aproximadamente igual a un precio que es una modificación nómica $F$ de la estructura productiva y del sistema de ponderación social del trabajo w:

$$
\mathbf{p} \approx F\left(\widetilde{\mathbf{y}}_{1}, \ldots, \tilde{\mathbf{y}}_{I}, \mathbf{w}\right),
$$

donde $F\left(\widetilde{\mathbf{y}}_{1}, \ldots, \widetilde{\mathbf{y}}_{I}, \mathbf{w}\right)$ es el precio inducido por $\mathbf{w}$. En el caso del modelo de Leontief,

$$
F\left(\widetilde{\mathbf{y}}_{1}, \ldots, \widetilde{\mathbf{y}}_{I}, \mathbf{w}\right)=[\rho \mathbf{I}-\underline{\mathbf{Y}}]^{-1} \cdot \mathbf{w} \mathbf{Y},
$$

pero la determinación de la forma específica de $F$ para otros estados de la economía puede resultar una tarea bastante difícil. Sin embargo, la meta del científico normal de la TVT es determinar la forma específica que asume la $F$. Esto sugiere que los términos explicativos de la TVT — TVT-teóricos_ - son precisamente alguna reducción estándar y la transformación $F$. La reducción estándar puede o no coincidir con el sistema salarial vigente, por lo que no debe ser identificada necesariamente con éste, que, a su vez, puede sufrir movimientos ocasionales de corto plazo. Así, los modelos potenciales podrían definirse como sigue.

DEFINICIÓN 11. M es una economía marxiana potencial syss existen $X_{i}, \succsim_{i}, Y_{j}, \boldsymbol{\omega}, \mathbf{e}, \mathbf{w}$ y $F$ tales que

(0) $\mathfrak{M}=\left\langle\left\langle X_{i}, \succsim_{i}\right\rangle, Y_{j}, \boldsymbol{\omega}, \mathbf{e}, \mathbf{w}, F\right\rangle$

(1) $\mathfrak{E}=\left\langle\left\langle X_{i}, \succsim_{i}\right\rangle, Y_{j}, \boldsymbol{\omega}\right\rangle$ es una economía;

(2) $\mathbf{e}=\left(\mathbf{x}_{1}, \ldots, \mathbf{x}_{I}, \mathbf{x}_{I+1}, \widetilde{\mathbf{y}}_{1}, \ldots, \widetilde{\mathbf{y}}_{J}, \mathbf{p}\right)$ es un estado de $\mathfrak{E}$

(3) w es una reducción estándar;

${ }^{12}$ Citado por Hamminga 1990, p. 92. 
(4) $F$ es una función de $\left\{\widetilde{\mathbf{y}}_{1}, \ldots, \widetilde{\mathbf{y}}_{J}, \mathbf{w}\right\}$ en el ortante no negativo de $\mathbb{R}^{L}$ tal que $F\left(\widetilde{\mathbf{y}}_{1}, \ldots, \widetilde{\mathbf{y}}_{J}, \mathbf{w}\right)$ es el sistema de precios inducido por $\mathbf{w}$.

La ley fundamental de la TVT - la ley del valor - permitiría definir los modelos del elemento teórico básico en los siguientes términos.

DEFINICIÓN 12. $\mathfrak{M}$ es una economía marxiana syss $\mathfrak{M}$ es una economía marxiana potencial que satisface la ley del valor:

$$
\mathbf{p} \approx F\left(\widetilde{\mathbf{y}}_{1}, \ldots, \widetilde{\mathbf{y}}_{J}, \mathbf{w}\right) .
$$

Queda así elucidada la aserción marxiana de que las proporciones de intercambio de las mercancías están determinadas, en la esfera de la producción, por el trabajo abstracto.

Grosso modo, así, lo que la TVT trata de explicar es un componente del estado vigente en la economía, el sistema de precios, en términos de otros componentes, los que conforman la estructura productiva, y algún sistema de salarios sombra. La forma general de la aserción empírica es que los precios vigentes en las economías capitalistas se pueden explicar de esta forma.

\section{BIBLIOGRAFÍA}

Álvarez, F., 1986, "Estructura y función de la ley del valor: un principio guía para la economía política”, en Álvarez, Broncano y Quintanilla 1986, pp. 374-389.

Álvarez, F., F. Broncano y M.A. Quintanilla (comps.), 1986, Filosofía e historia de la Ciencia, Ediciones Universidad de Salamanca/Excma. Diputación Provincial de Salamanca, Salamanca.

Arrow, K.J. y G. Debreu, 1954, "Existence of an Equilibrium for a Competitive Economy", Econometrica, vol. 22, no. 3, pp. 265-290.

Balzer, W., C.U. Moulines y J.D. Sneed, 1987, An Architectonic for Science, D. Reidel, Dordrecht.

Brzeziński, J., F. Coniglione, T.A.F. Kuipers y L. Nowak (comps.), 1990, Idealization I: General Problems. Poznan Studies in the Philosophy of the Sciences and the Humanities, vol. 16, Rodopi, Ámsterdam.

Brzeziński, J., A. Klawiter, T.A.F. Kuipers, K. Lastowski, K. Paprzycka y P. Przybysz, 2007, The Courage of Doing Philosophy. Essays Presented to Leszek Nowak, Rodopi, Ámsterdam/Nueva York.

* El presente artículo fue producido con el apoyo del proyecto CONACYT 127380 , Filosofía de la Economía. 
Debreu, G., 1973, Teoría del valor, Antoni Bosch, Barcelona.

Gale, D., 1960, The Theory of Linear Economic Models, The University of Chicago Press, Chicago/Londres.

García de la Sienra, A., 2010, "The Economic Sphere”, Axiomathes, vol. 20, pp. 81-94.

— , 2007, "Idealization in the Labor Theory of Value", en Brzeziński, Klawiter, Kuipers, Łastowski, Paprzycka y Przybysz 2007, pp. 219-233.

— 2003a, “Axiomas generales de la teoría del valor", en Uribe 2003, pp. 3-16.

——, 2003b, "Trabajo abstracto", en Uribe 2003, pp. 25-32.

- 1996, "La medición del trabajo abstracto", Economía mexicana, vol. 5 , no. 1 , pp. 63-75.

- 1992, The Logical Foundations of the Marxian Theory of Value, Kluwer Academic Publishers, Dordrecht.

- 1988, "Axiomatic Foundations of the Marxian Theory of Value", Erkenntnis, vol. 29, pp. 299-341.

— 1982, "The Basic Core of the Marxian Economic Theory", en Stegmüller, Balzer y Spohn 1982, pp. 118-144.

—, 1980 , "Elementos para una reconstrucción lógica de la teoría del valor de Marx", Crítica, vol. 12, no. 35, pp. 71-95.

Hamminga, B., 1990, "The Structure of Six Transformations in Marx's Capital", en Brzeziński, Coniglione, Kuipers y Nowak 1990, pp. 89-11l.

Krause, U., 1980, "Abstract Labour in General Joint Systems", Metroeconomica, vol. 32, nos. 2-3, pp. 115-135.

Marx, K., 1981, Capital, 3 vols., Penguin, Harmondsworth.

McCarthy, G.E., 1990, Marx and the Ancients. Classical Ethics, Social Justice, and Nineteenth-Century Political Economy, Rowman and Littlefield, Savage.

Morishima, M., y F. Seton, 1961, "Aggregation in Leontief Matrices and the Labour Theory of Value", Econometrica, vol. 29, no. 2, pp. 203-220.

Moulines, C.U., 1978, "Cuantificadores existenciales y principios guía en las teorías físicas", Crítica, vol. 29, pp. 59-88.

Okishio, N., 1963, “A Mathematical Note on Marxian Theorems", Weltwirtschaftliches Archiv, vol. 91, pp. 287-299.

Stegmüller, W., W. Balzer y W. Spohn (comps.), 1982, Philosophy of Economics, Springer-Verlag, Berlín/Heidelberg/Nueva York.

Takayama, A., 1985, Mathematical Economics, Cambridge University Press, Cambridge.

Uribe, P., 2003, La economía política del siglo XXI, Universidad de Guadalajara, Guadalajara.

Recibido el 1 de febrero de 2011; revisado el 18 de noviembre de 2011; aceptado el 8 de febrero de 2012. 